\title{
On monochromatic pairs with nondecreasing diameters
}

\author{
Adam O’Neal Michael W. Schroeder* \\ Department of Mathematics \\ Marshall University \\ Huntington, WV, U.S.A. \\ \{onleala, schroederm\}@marshall.edu
}

Submitted: Jul 14, 2018; Accepted: Apr 18, 2019; Published: May 3, 2019

(C) The authors. Released under the CC BY-ND license (International 4.0).

\begin{abstract}
Let $n, m, r, t$ be positive integers and $\Delta:[n] \rightarrow[r]$. We say $\Delta$ is $(m, r, t)-$ permissible if there exist $t$ disjoint $m$-sets $B_{1}, \ldots, B_{t}$ contained in $[n]$ for which

(a) $\left|\Delta\left(B_{i}\right)\right|=1$ for each $i=1,2, \ldots, t$,

(b) $\max \left(B_{i}\right)<\min \left(B_{i+1}\right)$ for each $i=1,2, \ldots, t-1$, and

(c) $\max \left(B_{i}\right)-\min \left(B_{i}\right) \leqslant \max \left(B_{i+1}\right)-\max \left(B_{i+1}\right)$ for each $i=1,2, \ldots, t-1$.

Let $f(m, r, t)$ be the smallest such $n$ so that all colorings $\Delta$ are $(m, r, t)$-permissible. In this paper, we show that $f(2,2, t)=5 t-4$.
\end{abstract}

Mathematics Subject Classifications: 05D10, 11B75, 11B50

\section{Introduction}

In this paper we study a Ramsey-type integer coloring problem posed by Bialostocki et al. [2] in 1995, which we restate using the definition of permissibility given below.

Definition 1. Let $n, m, r$, and $t$ be positive integers and $\Delta:[n] \rightarrow[r]$ be a coloring. We say $\Delta$ is $(m, r, t)$-permissible if there exists a collection of disjoint $m$-sets $\left\{B_{1}, B_{2}, \ldots, B_{t}\right\}$ each contained in $[n]$ such that

- $\left|\Delta\left(B_{i}\right)\right|=1$ for each $i=1,2, \ldots, t$,

- $\max \left(B_{i}\right)<\min \left(B_{i+1}\right)$ for each $i=1,2, \ldots, t-1$, and

- $\max \left(B_{i}\right)-\min \left(B_{i}\right) \leqslant \max \left(B_{i+1}\right)-\min \left(B_{i+1}\right)$ for each $i=1,2, \ldots, t-1$.

\footnotetext{
*Corresponding author
} 
We say the collection $\left\{B_{1}, B_{2}, \ldots, B_{t}\right\}$ is permissible in $\Delta$ if they satisfy the above conditions, thereby realizing $\Delta$ as $(m, r, t)$-permissible.

The first condition requires the $m$-sets be monochromatic; note two $m$-sets can be associated with different colors. The second condition is a non-overlapping property establishing precedence between the $m$-sets, and the third condition requires the ranges of each $m$-set, called their diameters, form a nondecreasing sequence. follows:

In this language the question posed by Bialostocki, Erdős, and Lefmann [2] is as

Question 2. Given positive integers $m, r$, and $t$, does there exist an integer $n$ such that for all $\Delta:[n] \rightarrow[r], \Delta$ is $(m, r, t)$-permissible? If so, what is the minimum possible value for $n$ ?

The answer to the first question is yes; it follows from a result of van der Waerden involving arithmetic progressions [11]. Bialostocki et al. define $f(m, r, t)$ as the smallest integer $n$ for which all $r$-colorings of $[n]$ are $(m, r, t)$-permissible. Several infinite families of parameters have $f(m, r, t)$ determined or bounded.

Observation 3. Note that for all positive integers $m, r$, and $t, f(m, r, t) \geqslant m t$ since the union of $t$ disjoint $m$-sets realizing a coloring as $(m, r, t)$-permissible must have cardinality no less than $f(m, r, t)$. This inequality is an equality if and only if $m=1$ or $r=1$. Additionally $f(m, r, 1)=(m-1) r+1$ by the pigeonhole principle.

Based on the above observation, interest in this question focuses on parameter families in which $m, r$, and $t$ are at least 2 .

Theorem 4 (Bialostocki et al. [2]). Let $m, r$, and $t$ be integers at least 2 .

(a) $f(m, 2,2)=5 m-3$.

(b) $f(m, 3,2)=9 m-7$.

(c) If $r \geqslant 4$, then

$$
3 r(m-1)+3 \leqslant f(m, r, 2)<((2 m-2)-r+1) \cdot\left(2+\log _{2} r\right)-1 .
$$

(d) $f(m, 2, t) \leqslant c m t^{2}$ for some constant $c$.

(e) $8 m-4 \leqslant f(m, 2,3) \leqslant 10 m-6$.

(f) $f(2, r, t) \leqslant(r(t-1)+1)(r+1)$.

Also in 1995 Bollobás, Erdős, and Jin [5] discovered results for the analogous question involving strictly increasing diameters, as well as established bounds for $f(2, r, 2)$ and gave asymptotic results for $f(2, r, t)$ : 
Theorem 5 (Bollobás et al. [5]).

(a) Let $r \geqslant 2$. Then $4 r-5 \log _{2} r<f(2, r, 2) \leqslant 4 r+1$.

(b) For a fixed $t, f(2, r, t)$ is linear in $r$.

In 2005 Grynkiewicz showed the lower bound of the inequality in Theorem 4(c) is an equality when $r=4$.

Theorem 6 (Grynkiewicz [9]). Let $m$ be a positive integer at least 2. Then

$$
f(m, 4,2)=12 m-9 .
$$

In 2015 Bernstein et al. found the value of $f(m, 2,3)$, first bounded in Theorem 4(e).

Theorem 7 (Bernstein et al. [1]). Let $m$ be a positive integer at least 2. Then

$$
f(m, 2,3)=8 m-5+\left\lfloor\frac{2 m-2}{3}\right\rfloor+\delta,
$$

where $\delta=1$ if $m \in\{2,5\}$ and $\delta=0$ otherwise.

Recent work has been done on generalizations of Question 2, as well as investigations into its relationship to a theorem by Erdős, Ginzburg, and Ziv (see [3, 4, 6, 7, 8, 10] for examples). With possibly the only exceptions being the bounds given in Theorem 4(d) and (f), as well as Theorem 5(b), all recent work in evaluating $f(m, r, t)$ focuses on parameter families with $t=2$ and more recently $t=3$ [1]. In our work we compute values for $f(2,2, t)$ for arbitrarily large $t$ which, as far as we can tell, is the first known set of actual values of $f(m, r, t)$ with $m, r \geqslant 2$ and arbitrarily large $t$, rather than asymptotic results.

Note that $f(2,2, t)$ has been computed when $t \leqslant 3 ; f(2,2,1)=3$ by Observation $3, f(2,2,2)=7$ by Theorem $4(\mathrm{a})$, and $f(2,2,3)=12$ by Theorem 7 . Additionally, $f(2,2, t) \leqslant 6 t-3$ by Theorem $4(\mathrm{f})$. Note that the upper bound given in Theorem $4(\mathrm{f})$ is not an attempt at a sharp bound. The inequality is found by using the pigeonhole principle and, in fact, guarantees the existence of a permissible collection of $t 2$-sets which all have the same diameter.

In this paper we prove the following:

Theorem 8. Let $t \geqslant 4$ be a positive integer. Then $f(2,2, t)=5 t-4$.

In Section 2 we introduce notation for the problem and present some constructions which are useful in later proofs for finding collections of permissible sets. In Section 3 we first prove that $f(2,2, t) \geqslant 5 t-4$ by presenting a coloring of $[5 t-5]$ which is not $(2,2, t)$ permissible, then prove a weak upper bound on $f(2,2, t)$ which gives the existence of a permissible collection of sets with additional structure. We finish the section with a proof that all colorings of [ $5 t-4]$ with $t \geqslant 4$ are $(2,2, t)$-permissible by showing there does not exist a coloring of $[5 t-4]$ which is not $(2,2, t)$-permissible. 


\section{Definitions and Constructions}

We begin this section with a series of definitions, terminology, and notation related to colorings with 2 colors (using the color set $\{a, b\}$ rather than [2]) and the identification of collections of 2 -sets (abbreviated pairs) which are permissible.

Let $n$ be a positive integer and $\Delta:[n] \rightarrow\{a, b\}$. At times we present $\Delta$ as the string $x_{1} x_{2} \cdots x_{n}$, where $x_{i}=\Delta(i)$ for each $i \in[n]$. We say $x_{i} x_{i+1} \cdots x_{i+k-1}$, where $x_{j}=\Delta(j)$ for each $j=i, i+1, \ldots, i+k-1$, is a string of length $k$ in $\Delta$. For $x \in\{a, b\}$, we use the abbreviation $x^{n}$ to denote the string $x x \cdots x$ ( $n$ times) and similarly define $w^{n}=w w \cdots w$ ( $n$ times), where $w$ is any word from the alphabet $\{a, b\}$. We say $\{i, i+1, \ldots, i+k-1\}$ is a $k$-tuple in $\Delta$ if $\Delta(i)=\Delta(i+1)=\cdots=\Delta(i+k-1)$. If $\Delta(i-1) \neq \Delta(i)$ (or $i=1$ ) and $\Delta(i+k-1) \neq \Delta(i+k)$ (or $i+k-1=n$ ), we say the $k$-tuple is isolated. Specifically, a double is a 2 -tuple and a triple is a 3 -tuple. A string $x_{i} x_{i+1} \cdots x_{i+k-1}$ in $\Delta$ of length $k$ is alternating if $\Delta(j) \neq \Delta(j+1)$ for each $j=i, i+1, \ldots, i+k-2$. A pair $\{i, i+k\}$ in $[n]$ is a monochromatic pair of diameter $k$, abbreviated $\mathrm{D}_{k}$, in $\Delta$ if $\Delta(i)=\Delta(i+k)$. Note that a $D_{1}$ is a double and a triple contains two $D_{1}$ s and a $D_{2}$.

We now define the alternating substring/triples partition of $[n]$ with respect to $\Delta$ (abbreviated the AST partition of $\Delta$ ) and give the process for constructing it. As we see later, this partition yields a permissible collection of pairs which have diameters 1 or 2 , which is instrumental going forward.

Construction 9. Let $n>0$ and $\Delta:[n] \rightarrow\{a, b\}$. By the following steps, we partition $[n]$ into alternating substrings and triples.

1. Find the maximum number of pairwise disjoint triples in $\Delta$. Suppose that there are $w$ of them; call them $T_{1}, T_{2}, \ldots, T_{w}$, and suppose the minimal element in $T_{i}$ is $\tau_{i}$ for each $i \in[w]$. We additionally require that for all $i \in[w]$, if $\tau_{i}>1$, then either $\Delta\left(\tau_{i}-1\right) \neq \Delta\left(\tau_{i}\right)$ or $\tau_{i}-1 \in T_{i-1}$. This ensures that we "frontload" the triples. In other words, we read through the string from left to right and define our triples in a greedy method. We define the collection of triples as $\mathcal{T}:=\left\{T_{1}, T_{2}, \ldots, T_{w}\right\}$.

2. Let $\mathcal{U}=\left\{U_{0}, U_{1}, \ldots, U_{w}\right\}$ be a collection of subsets of $[n]$ defined as $U_{0}=\left[\min \left(T_{1}\right)-1\right]$, $U_{i}=\left[\max \left(T_{i}\right)+1, \min \left(T_{i+1}\right)-1\right]$ for each $i=1,2, \ldots, w-1$, and $U_{w}=\left[\max \left(T_{w}\right)+1, n\right]$. Note that some of these sets may be empty if $\Delta$ contains consecutive triples, or begins or ends with a triple. It follows that $\mathcal{U} \cup \mathcal{T}$ is a partition of $[n]$.

3. Partition each $U_{i}$ into consecutive alternating substrings of maximal length. Note that if there are $s$ such maximal substrings in $U_{i}$, then there will be $s-1$ doubles contained in $U_{i}$. Thus in total we have $v+w+1$ such maximal alternating substrings $S_{i}$, $i \in[v+w+1]$, where we use the convention that an empty set $U_{i}$ corresponds to an empty substring. Thus the substrings $S_{i}$ partition the terms of $\bigcup_{i=0}^{w} U_{i}$ as $\bigcup_{i=1}^{v+w+1} S_{i}$. Let $\mathcal{S}=\left\{S_{1}, S_{2}, \ldots, S_{v+w+1}\right\}$ be the collection of these alternating substrings, and for each $i \in[v+w+1]$, let $k_{i}=\left|S_{i}\right|$ be the length of $S_{i}$.

Observe that $\mathcal{S} \cup \mathcal{T}$ is a partition of $[n]$, and so we define $(\mathcal{S}, \mathcal{T} ; v, w)$ as the AST partition of $\Delta$. Since $\mathcal{S} \cup \mathcal{T}$ is a partition of $[n]$, note $n=3 w+k_{1}+\cdots+k_{v+w+1}$. 
Example 10. Let $\Delta_{1}:[16] \rightarrow\{a, b\}$ be given by abaaababaabaaaba. Then the AST partition of $\Delta_{1}$ has $v=1$ and $w=2$ with $\mathcal{T}=\{\{3,4,5\},\{12,13,14\}\}$ and $\mathcal{S}=$ $\{\{1,2\},\{6,7,8,9\},\{10,11\},\{15,16\}\}$. One can see this visually as

$$
\Delta_{1}: \frac{a b}{S_{1}} \frac{a a a}{T_{1}} \frac{b a b a}{S_{2}} \frac{a b}{S_{3}} \frac{a a a}{T_{2}} \frac{b a}{S_{4}}
$$

Let $\Delta_{2}:[36] \rightarrow\{a, b\}$ be given as abababababaababaaabaabbababbbaaaaab. Then the AST partition of $\Delta_{2}$ has $v=4$ and $w=3$ with the parts indicated below.

$$
\Delta_{2}: \frac{a b a b a b a b a b a}{S_{1}} \frac{a b a b}{S_{2}} \frac{a a a}{T_{1}} \frac{b a}{S_{3}} \frac{a b}{S_{4}} \frac{b a b a}{S_{5}} \frac{b b b}{T_{2}} \frac{a a a}{S_{6}} \frac{a}{T_{3}} \frac{a b}{S_{7}}
$$

Definition 11. Let $(\mathcal{S}, \mathcal{T} ; v, w)$ be the AST partition of $\Delta:[n] \rightarrow\{a, b\}$ for some positive integer $n$. Observe that $\mathcal{S} \cup \mathcal{T}$ is a partition of $[n]$ into sets of consecutive integers, and these $v+2 w+1$ parts have an implied order. With this in mind, we say $\Delta$ is of type $\gamma$, where $\gamma=\left(\gamma_{1}, \gamma_{2}, \ldots, \gamma_{v+2 w+1}\right)$ is a $(v+2 w+1)$-tuple, which is an ordering of $k_{1}, k_{2}, \ldots, k_{v+w+1}$ and $w$ copies of the symbol $\tau$ where the order of the ordinates reflects the relative order of the alternating substrings and triples in the AST partition of $\Delta$. We say $\Delta$ contains $\mathbf{y}=\left(y_{1}, y_{2}, \ldots, y_{\ell}\right)$ for some $\ell$ and symbols $y_{i}, i \in[\ell]$ if, for some $j, y_{i}=\gamma_{i+j}$ for each $i \in[\ell]$. Specifically, we say $\Delta$ ends with $\mathbf{y}$ if $\Delta$ contains $\mathbf{y}$ and $j=v+2 w+1-\ell$. For convenience, we use $\bar{a}$ to mean "is congruent to $a \bmod 3$ and at least $a$ ".

Example 12. Let $\Delta_{1}$ and $\Delta_{2}$ be the colorings from Example 10. Then $\Delta_{1}$ is type $(2, \tau, 4,2, \tau, 2)$ and $\Delta_{2}$ is type $(11,4, \tau, 2,2,4, \tau, 0, \tau, 1,2)$. We may also say $\Delta_{1}$ contains $(\tau, \overline{1}, 2, \tau)$ and ends with $(\tau, 2)$, while $\Delta_{2}$ contains $(\overline{1}, \tau, 2)$ and ends with $(\tau, \overline{1}, \overline{2})$.

Observation 13. Let $(\mathcal{S}, \mathcal{T} ; v, w)$ be the AST partition of $\Delta:[n] \rightarrow\{a, b\}$. If $v+w \geqslant t$, then $\Delta$ contains $w$ disjoint triples - each of which contain a double - and $v$ disjoint doubles which are disjoint from the triples. Therefore $\Delta$ contains $v+w \geqslant t$ disjoint $\mathrm{D}_{1} \mathrm{~s}$, which implies $\Delta$ is $(2,2, t)$-permissible.

Observation 14. Let $k>0$ and $w:[k] \rightarrow\{a, b\}$ be a coloring represented by an alternating string, i.e. $w=a b a b \ldots a$ if $k$ is odd and $w=a b a b \cdots a b$ if $k$ is even. Then $\{\{3 i-2,3 i\} \mid i=1, \ldots,\lfloor k / 3\rfloor\}$ is a permissible collection of $\lfloor k / 3\rfloor \mathrm{D}_{2} \mathrm{~s}$. In this manner, all alternating strings of length $k$ contain a permissible collection of $\lfloor k / 3\rfloor \mathrm{D}_{2} \mathrm{~s}$.

Definition 15. Let $\Delta:[n] \rightarrow\{a, b\}$ with AST partition $(\mathcal{S}, \mathcal{T} ; v, w)$. Each of the $w$ triples in $\mathcal{T}$ contain a $D_{2}$, while each alternating substring in $\mathcal{S}$ contains a permissible collection of $\lfloor k / 3\rfloor \mathrm{D}_{2}$ s. Hence $\Delta$ has a permissible collection of $w+\left\lfloor k_{1} / 3\right\rfloor+\cdots+\left\lfloor k_{v+w+1} / 3\right\rfloor \mathrm{D}_{2} \mathrm{~s}$. As previously indicated in Observation 14, we "frontload" when selecting $\mathrm{D}_{2} \mathrm{~S}$ from an alternating substring by reading from left to right. We call this collection of $\mathrm{D}_{2} \mathrm{~s}$ of $\Delta$ the canonical $\mathrm{D}_{2} \mathrm{~S}$ of $\Delta$.

Example 16. Let $\Delta_{1}$ and $\Delta_{2}$ be the colorings defined in Examples 10 and 12 . There are 3 canonical $D_{2} \mathrm{~S}$ in $\Delta_{1}$, which are $\{3,5\},\{6,8\}$, and $\{12,14\}$, seen below:

$$
\Delta_{1}: \frac{a b}{S_{1}} \frac{a \propto a}{T_{1}} \frac{b a b a}{S_{2}} \frac{a b}{S_{3}} \frac{a a a}{T_{2}} \frac{b a}{S_{4}}
$$


In a similar manner there are 8 canonical $D_{2} \mathrm{~s}$ in $\Delta_{2}$, which are illustrated below.

$$
\Delta_{2}: \frac{a b a b a b a b a b a}{S_{1}} \frac{a b a b}{S_{2}} \frac{a a a}{T_{1}} \frac{b a}{S_{3}} \frac{a b}{S_{4}} \frac{b a b a}{S_{5}} \frac{b b b}{T_{2}} \frac{a a a}{S_{6}} \frac{a}{T_{3}} \frac{a b}{S_{7}}
$$

\section{Main Results}

We prove Theorem 8 in two stages. First, we give what will be the sharp lower bound on $f(2,2, t)$, then produce a weaker upper bound by showing when $n$ is large enough, there is a permissible collection of pairs with bounded diameters contained in any 2-coloring of $[n]$. We conclude the section by establishing properties that a non-permissible coloring of $[5 t-4]$ must have, then showing that no coloring satisfies all of the properties.

\subsection{Bounds on $f(2,2, t)$}

We first show that $f(2,2, t) \geqslant 5 t-4$ by demonstrating the existence of a coloring of $[5 t-5]$ which is not $(2,2, t)$-permissible. This is derived by setting $m=r=2$ in the following theorem.

Theorem 17. Let $m, r, t \geqslant 2$ and $\Delta$ be the $r$-coloring of $[(m r+1)(t-1)]$ given by $\left((12 \cdots r)^{m} 1\right)^{t-1}$. Then $\Delta$ is not $(m, r, t)$-permissible.

Proof. The result is trivial for $t=2$. Assume that $\left((12 \cdots r)^{m} 1\right)^{t-2}$ is not $(m, r, t-1)$ permissible for some $t \geqslant 3$ and $\left((12 \cdots r)^{m} 1\right)^{t-1}$ is $(m, r, t)$-permissible. Let $\left\{B_{1}, \ldots, B_{t}\right\}$ be a permissible collection of pairs in $\left((12 \cdots r)^{m} 1\right)^{t-1}$.

Since $\left((12 \cdots r)^{m} 1\right)^{t-2}$ is not $(m, r, t-1)$-permissible, we have $\min \left(B_{2}\right) \leqslant m r+1$; otherwise $\left\{B_{2}, \ldots, B_{t}\right\}$ is a permissible collection of $t-1$ pairs in $\Delta$ restricted to $[m r+$ $2,(m r+1)(t-1)]$, implying that $\left((12 \cdots r)^{m} 1\right)^{t-2}$ is $(m, r, t-1)$-permissible. Hence $B_{1}=\{i, r+i, \ldots,(m-1) r+i\}$ for some $i \in[r]$, and thus $\operatorname{diam}\left(B_{1}\right)=m(r-1)$. Similarly, we have that $\max \left(B_{t-1}\right)>(r m+1)(t-2)$ and hence $\operatorname{diam}\left(B_{t}\right)=r(m-1)$ for reasons identical to those above,. Therefore $\operatorname{diam}\left(B_{2}\right)=r(m-1)$, and it follows that if $\min \left(B_{2}\right)=j$ for some $j \in[r]$ (and necessarily $r(m-1)+2 \leqslant \min \left(B_{2}\right) \leqslant m r+1$ ):

$$
\Delta\left(\max \left(B_{2}\right)\right)=\Delta\left(\min \left(B_{2}\right)+r(m-1)\right)= \begin{cases}j-1 & \text { if } j \neq 1 \text { and } \\ r & \text { if } j=1\end{cases}
$$

Thus $B_{2}$ is not monochromatic, giving a contradiction. So $\left((12 \cdots r)^{m} 1\right)^{t-1}$ is not $(m, r, t)-$ permissible. It follows that $f(2,2, t)>5 t-5$.

We now show that $f(2,2, t) \leqslant 5 t-2$, a weaker result than what Theorem 8 would guarantee, but we show this bound can be realized by a permissible collection of pairs with bounded diameter.

Theorem 18. Let $t \geqslant 1$ be an integer and $\Delta:[5 t-2] \rightarrow\{a, b\}$. Then $\Delta$ is $(2,2, t)$ permissible, and there is a permissible collection of $t$ pairs having diameter at most 2 . 
Proof. Let $(\mathcal{S}, \mathcal{T} ; v, w)$ be the AST partition of $\Delta$. By Observation 13 if $v+w \geqslant t$, then $\Delta$ is $t$-permissible as it contains at least $t$ disjoint $\mathrm{D}_{1}$ s. Now suppose $v+w \leqslant t-1$. Recall that for each $i \in[v+w+1], k_{i}=\left|S_{i}\right|$, and define $k_{i}^{\prime} \in\{0,1,2\}$ so that $k_{i}^{\prime}=k_{i} \bmod 3$. Note $\left(k_{i}-k_{i}^{\prime}\right) / 3=\left\lfloor k_{i} / 3\right\rfloor$ for each $i \in[v+w+1]$. Let $\sigma$ denote the number of canonical $\mathrm{D}_{2} \mathrm{~s}$ of $\Delta$. Recall $5 t-2=3 w+k_{1}+k_{2}+\cdots+k_{v+w+1}$ and $\sigma=w+\left\lfloor k_{1} / 3\right\rfloor+\left\lfloor k_{2} / 3\right\rfloor+\cdots+\left\lfloor k_{v+w+1} / 3\right\rfloor$. So

$$
\begin{aligned}
3 \sigma & =3 w+\left(k_{1}-k_{1}^{\prime}\right)+\left(k_{2}-k_{2}^{\prime}\right)+\cdots+\left(k_{v+w+1}-k_{v+w+1}^{\prime}\right) \\
& =(5 t-2)-\left(k_{1}^{\prime}+k_{2}^{\prime}+\cdots+k_{v+w+1}^{\prime}\right) \\
& \geqslant 5 t-2-2(v+w+1) \\
& \geqslant 5 t-2-2 t=3 t-2 .
\end{aligned}
$$

So $\sigma \geqslant t-\frac{2}{3}$, and since $\sigma$ and $t$ are integers, $\sigma \geqslant t$. By choosing any $t$ of the $\sigma$ canonical $\mathrm{D}_{2} \mathrm{~S}$ of $\Delta$, we have a permissible collection of $t$ pairs in $\Delta$. So $\Delta$ is $(2,2, t)$-permissible and is realized by a permissible collection of pairs with diameter at most 2 .

\subsection{Colorings of $[5 t-4]$ which are not $(2,2, t)$-permissible}

For the remainder of this section, let $\Delta$ be a coloring of $[5 t-4],(\mathcal{S}, \mathcal{T} ; v, w)$ be the AST partition of $\Delta, \mathcal{D}$ be the set of canonical $\mathrm{D}_{2} \mathrm{~s}$ of $\Delta$, and $\sigma=|\mathcal{D}|$. We now outline a series of properties that $\Delta$ must satisfy to not be $(2,2, t)$-permissible. We conclude this section by showing no coloring can satisfy all such conditions, which establishes $f(2,2, t) \leqslant 5 t-4$, and thus proves Theorem 8 .

As it will be relevant in the proof of Theorem 8, take note that all of the following lemmas and observations are valid for $t \geqslant 2$, and we indicate where appropriate what the largest diameter for a realization of permissibility is for a coloring. This will be leveraged in the final proof. We begin an observation about the number of canonical $\mathrm{D}_{2} \mathrm{~S}$ in $\Delta$ provided it is not $(2,2, t)$-permissible.

Observation 19. Let $t \geqslant 2$ and suppose $\Delta$ is not $(2,2, t)$-permissible. By a similar argument found in (1), we find $3 \sigma \geqslant 5 t-4-2(v+w+1)$. So $\sigma \geqslant t-\frac{4}{3}$ and hence $\sigma \geqslant t-1$. By Observation 13, we have $\sigma=t-1$, and hence $\Delta$ contains exactly $t-1$ canonical $\mathrm{D}_{2} \mathrm{~S}$.

The previous observation allows us to classify the value of $v+w$ in the AST partition of $\Delta$ given it is not $(2,2, t)$-permissible.

Lemma 20. Let $t \geqslant 2$. If $\Delta$ is not $(2,2, t)$-permissible, then $v+w=t-1$. Therefore $\Delta$ has exactly $t$ alternating substrings in its AST partition.

Proof. By Observation 13, $v+w \leqslant t-1$. Suppose $v+w \leqslant t-2$. Then in a manner similar to what is shown in (1) and Observation 19, we find

$$
3 \sigma \geqslant 5 t-4-2(v+w+1) \geqslant 5 t-4-2(t-2)=3 t .
$$

So $\sigma \geqslant t$ and therefore $\Delta$ contains at least $t$ canonical $\mathrm{D}_{2}$ s. Hence $\Delta$ is $(2,2, t)$-permissible with a realization whose largest diameter is 2 . 
Adapting the proof for Theorem 18 allows us to determine the congruence for $\left|S_{i}\right|$ for each $i=1,2, \ldots, v+w+1$.

Lemma 21. Let $t \geqslant 2$. If $\Delta$ is not $(2,2, t)$-permissible, there exists $j \in\{1,2, \ldots, v+w+1\}$ such that $\left|S_{j}\right| \equiv 1 \bmod 3$ and $\left|S_{k}\right| \equiv 2 \bmod 3$ whenever $k \neq j, 1 \leqslant k \leqslant v+w+1$.

Proof. By Lemma 20, we have $t=v+w+1$ and therefore $\Delta$ has $t$ alternating substrings in its AST partition. Again for each $i \in[t]$, define $k_{i}^{\prime} \in\{0,1,2\}$ so that $k_{i}^{\prime} \equiv k_{i} \bmod 3$. By Observation 19 we have $\sigma=t-1$. Combining this with a similar computation to (1) we get

$$
3(t-1)=3 \sigma=3 w+\sum_{i=1}^{t}\left(k_{i}-k_{i}^{\prime}\right)=3 w+\sum_{i=1}^{t} k_{i}-\sum_{i=1}^{t} k_{i}^{\prime}=5 t-4-\sum_{i=1}^{t} k_{i}^{\prime} .
$$

So $k_{1}^{\prime}+\cdots+k_{t}^{\prime}=2 t-1$. Therefore exactly one term in this sum is 1 while the remaining terms are each 2 . Hence $\Delta$ is $(2,2, t)$-permissible with a realization whose largest diameter is 2 .

We now classify the $k$-tuples which may exist in $\Delta$.

Lemma 22. If $\Delta$ is not $(2,2, t)$-permissible, then $\Delta$ does not contain an isolated 4-tuple or isolated $k$-tuple for each $k \geqslant 6$.

Proof. First, observe that if $\Delta$ contains a $k$-tuple for some $k \geqslant 6$, then $\Delta$ has at least two consecutive triples in its AST partition. Hence there is an empty substring $S_{i} \in \mathcal{S}$ for some $i \in[v+w+1]$, giving that $k_{i} \equiv 0 \bmod 3$. So $\Delta$ is $(2,2, t)$-permissible by Lemma 21 with a realization whose largest diameter is $2-$ a contradiction.

Now suppose $\Delta$ contains the isolated 4-tuple $\{\ell, \ell+1, \ell+2, \ell+3\}$ for some $\ell \in$ $[n-3]$. For each $i \in[w]$, let $T_{i}^{\prime}$ be the set containing the smallest two elements in $T_{i}$. It follows from Construction 9 that $T_{j}=\{\ell, \ell+1, \ell+2\}$ for some $j \in\{1,2, \ldots, w\}$ and $\ell+3$ does not belong to any double or triple identified in the AST partition. Then $\left\{P_{1}, P_{2}, \ldots, P_{v}, T_{1}^{\prime}, T_{2}^{\prime}, \ldots, T_{w}^{\prime},\{\ell+2, \ell+3\}\right\}$ is a permissible collection of $v+w+1$ doubles, and since $v+w+1=t$ by Lemma $20, \Delta$ is $(2,2, t)$-permissible with a realization whose largest diameter is 1 - a contradiction.

With the previous two lemmas, we now establish that $\Delta$ can have at most one triple and, if $\Delta$ contains a triple, give conditions on the lengths of the alternating substrings surrounding the triple.

Lemma 23. If $\Delta$ is not $(2,2, t)$-permissible and $\Delta$ contains any triples, then $\Delta$ contains $(\overline{1}, \tau, \overline{2})$ or $(\overline{2}, \tau, \overline{1})$. Furthermore $\Delta$ contains at most one triple.

Proof. Observe that $\Delta$ cannot begin with a triple, end with a triple, or have two consecutive triples in its AST partition; otherwise one of its alternating substrings has length 0, which contradicts Lemma 21. Therefore every triple in the AST partition of $\Delta$ is preceded and followed by a nonempty alternating substring. Furthermore by Lemma 21, it follows 
that if $\Delta$ contains a triple in its AST partition, then $\Delta$ must contain $(\overline{1}, \tau, \overline{2}),(\overline{2}, \tau, \overline{1})$, or $(\overline{2}, \tau, \overline{2})$.

Assume $\Delta$ contains $(x, \tau, y)$, where $x \equiv y \equiv 2 \bmod 3$, and let $S_{L}, T$, and $S_{R}$ denote the corresponding alternating substrings and triple, and let $i$ be the integer such that $T=\{i, i+1, i+2\}$. Since $\Delta(i-2) \neq \Delta(i-1)$, it following by the AST construction that $\Delta(i-1) \neq \Delta(i)$. Since $\Delta(i+3) \neq \Delta(i+4)$, it follows by Lemma 22 that $\Delta(i+2) \neq \Delta(i+3)$. Thus $S_{L} \cup\{i\}$ and $S_{R} \cup\{i+2\}$ are alternating substrings of lengths $x+1$ and $y+1$, respectively. Hence $S_{L} \cup\{i\}$ contains a permissible collection of $\lfloor x / 3\rfloor+1 \mathrm{D}_{2} \mathrm{~S}$, and similarly $S_{R} \cup\{i+2\}$ contains a permissible collection of $\lfloor y / 3\rfloor+1 \mathrm{D}_{2} \mathrm{~s}$. Removing those $\mathrm{D}_{2}$ s from $\mathcal{D}$ contained in $S_{L}, S_{R}$, and $T$, then combining them with the permissible collections of $\mathrm{D}_{2} \mathrm{~S}$ contained in $S_{L} \cup\{i\}$ and $S_{R} \cup\{i+2\}$ produces a permissible collection of $t \mathrm{D}_{2} \mathrm{~s}$, which is a contradiction. So $\Delta$ does not contain $(\overline{2}, \tau, \overline{2})$. So if $\Delta$ contains any triples, then $\Delta$ contains $(\overline{2}, \tau, \overline{1})$ or $(\overline{1}, \tau, \overline{2})$.

By Lemma 21, there is exactly one substring in the AST partition of $\Delta$ which has length congruent to $1 \bmod 3$. Therefore $w \leqslant 2$, and if $w=2$ then $\Delta$ must contain $(\overline{2}, \tau, \overline{1}, \tau, \overline{2})$. Assume that $w=2$ and $\Delta$ contains $(x, \tau, y, \tau, z)$, where $y \equiv 1 \bmod 3$ and $x \equiv z \equiv 2 \bmod 3$. Let $S_{L},\{i, i+1, i+2\}, S_{M},\{j, j+1, j+2\}, S_{R}$ be the associated alternating substrings and triples. In a manner similar to the previous argument, $S_{L} \cup$ $\{i\},\{i+2\} \cup S_{M} \cup\{j\}$, and $\{j+2\} \cup S_{R}$ are alternating substrings. By removing the $\mathrm{D}_{2} \mathrm{~S}$ in $\mathcal{D}$ contained in $S_{L} \cup\{i, i+1, i+2\} \cup S_{M} \cup\{j, j+1, j+2\} \cup S_{R}$ and adding those contained in $S_{L} \cup\{i\},\{i+2\} \cup S_{M} \cup\{j\}$, and $\{j+2\} \cup S_{R}$, we produce a permissible collection of $t \mathrm{D}_{2} \mathrm{~S}$ in $\Delta-$ a contradiction. Thus $w \leqslant 1$.

Note that in each of the above alterations, each coloring was found to be $(2,2, t)$ permissible with a realization whose largest diameter is 2 .

We now give an observation which classifies the possible final substring in $\Delta$ and is useful in the arguments which follow.

Observation 24. Suppose $\Delta$ is not $(2,2, t)$-permissible.

(a) $\Delta(5 t-4) \neq \Delta(5 t-6)$. Otherwise $\left.\Delta\right|_{[5 t-7]}$ is a coloring which is $(2,2, t-1)$-permissible with a permissible collection of $(t-1)$ pairs of diameter at most 2 by Theorem 18 . The collection of these $(t-1)$ sets with $\{5 t-6,5 t-4\}$ yields a permissible collection of $t$ pairs in $\Delta$ with a largest diameter of 2 .

With this in mind, since $\Delta$ cannot end with a triple, the alternating substring which ends $\Delta$ cannot have length exceeding 2. So $k_{v+w+1}=1$ or $k_{v+w+1}=2$ and thus $\Delta$ must end with type (1) or (2).

(b) $\Delta(5 t-11) \neq \Delta(5 t-9)$. Otherwise $\left.\Delta\right|_{[5 t-9]}$ is $(2,2, t-1)$-permissible with a permissible collection of $t-1$ pairs whose largest diameter is at most 2 . Since either $\{5 t-8,5 t-6\}$, $\{5 t-8,5 t-4\}$, or $\{5 t-6,5 t-4\}$ is a monochromatic pair, we can produce a permissible collection of $t$ pairs, which means that $\Delta$ is $(2,2, t)$-permissible, and this realization has a largest diameter of either 2 or 4 .

Now we give a proof for the main result. 
Proof of Theorem 8. Suppose $\Delta$ is not $(2,2, t)$-permissible. By Observation 24(a), $\Delta$ must end with type (1) or (2). By Lemma 23, $\Delta$ may contain at most 1 triple and if $\Delta$ contains a triple, then $(\overline{2}, \tau, \overline{1})$ or $(\overline{1}, \tau, \overline{2})$ is contained in $\Delta$. That said there are 12 types with which $\Delta$ may end; see Figure 1.

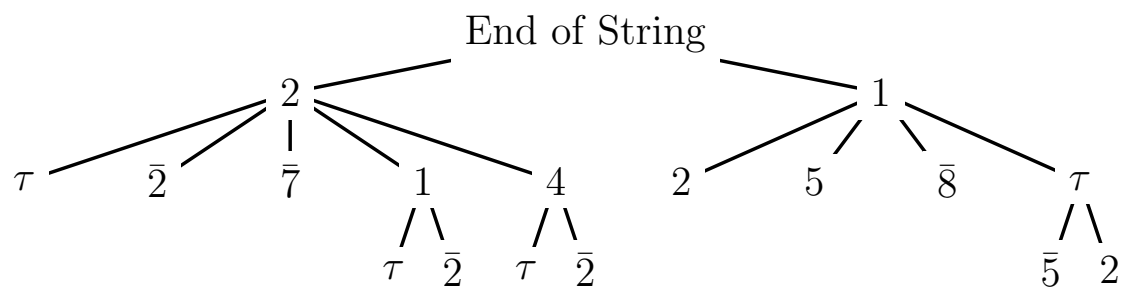

Figure 1: The possible endings for a coloring which is not $(2,2, t)$-permissible. For example, the left-most leaf corresponds to type $(\tau, 2)$, while the right-most leaf corresponds to type $(2, \tau, 1)$.

We now show in each case above that $\Delta$ is, in fact, $(2,2, t)$-permissible and therefore prove Theorem 8 . Without loss of generality, we assume $\Delta(5 t-4)=b$. Suppose $\Delta$ ends with type:

- $(\tau, 2)$. Since $\Delta$ cannot end with bbbab by Observation $24(\mathrm{a})$, we have $\Delta$ ends with aaaab. Since $t \geqslant 2$, there is a nonempty alternating substring $S_{t-1}$ which precedes the triple. If the last letter in $S_{t-1}$ is an $a$, then by the AST construction $\Delta$ has two consecutive triples, which violates Lemma 23. So $\Delta$ ends with baaaab, in which case we have an isolated quadruple in $\Delta$, which contradicts Lemma 22. Thus a coloring with this end type is $(2,2, t)$-permissible with a realization having a largest diameter at most 2 .

- $(\overline{2}, 2)$. Then $\Delta$ ends with baab, and all of the corresponding indices in $[5 t-7,5 t-4]$ are preceded by the $t-1$ canonical $\mathrm{D}_{2} \mathrm{~s}$ of $\Delta$. Then $\mathcal{D} \cup\{\{5 t-7,5 t-4\}\}$ is a permissible collection of $t$ pairs and hence $\Delta$ is $(2,2, t)$-permissible with a realization whose largest diameter is 3 .

- $(\overline{7}, 2),(\overline{8}, 1)$, or $(\overline{5}, \tau, 1)$. By Observation $24(\mathrm{~b}), \Delta$ is $(2,2, t)$-permissible with a realization having a largest diameter at most 4 . Note this argument implies that, if $\Delta$ is a coloring of [5t-4] with $t \geqslant 3$ (rather than 4 ) and ending with any of these three types, then $\Delta$ is also $(2,2, t)$-permissible. This will be relevant in the last subcase of this proof.

- $(\tau, 1,2)$. Since $t \geqslant 3$, by Lemmas 21 and $23, \Delta$ ends with type $(\overline{2}, \tau, 1,2)$. So $\Delta$ ends with either abaaaaab, baaaaaab, babbbaab, or abbbbaab. Then $\Delta$ is $(2,2, t)$ permissible by Observation 24(b) for the first or third case and by Lemma 22 for the second or fourth case. Thus $\Delta$ is $(2,2, t)$-permissible with a realization having a largest diameter at most 4 . 
- $(\overline{2}, 1,2)$. In this case $\Delta$ ends with baaab, meaning the triples were not correctly identified in its AST partition. So $\Delta$ cannot end with this type.

- $(\tau, 4,2)$. Since $t \geqslant 3$, by Lemmas 21 and $23, \Delta$ must end with type $(\overline{2}, \tau, 4,2)$. Then $\Delta$ ends with abaaababaab, abbbbbabaab, baaaababaab, or babbbbabaab. Note the second and third cases are not possible as the triples were not selected properly for its partition. In the first case, $\{5 t-12,5 t-10\}$ and $\{5 t-9,5 t-7\}$ belong to $\mathcal{D}$. The collection the pairs in $\mathcal{D}$, excluding $\{5 t-12,5 t-10\}$ and $\{5 t-9,5 t-7\}$, combined with $\{5 t-14,5 t-12\},\{5 t-11,5 t-8\}$, and $\{5 t-7,5 t-4\}$ is permissible, demonstrating that $\Delta$ is $(2,2, t)$-permissible. Finally $\Delta$ is $(2,2, t)$-permissible by Observation 24(b) for the fourth case. Thus $\Delta$ is $(2,2, t)$-permissible with a realization having a largest diameter at most 4 .

- $(\overline{2}, 4,2)$ or $(5,1)$. In the latter case, since $t \geqslant 3$, by Lemmas 21 and $23, \Delta$ must end with type $(\overline{2}, 5,1)$. So $\Delta$ ends with abbabaab or abbababb. In a similar manner to the above case, the collection of pairs in $\mathcal{D}$, excluding $\{5 t-9,5 t-7\}$, combined with $\{5 t-11,5 t-8\}$ and $\{5 t-7,5 t-4\}$ is permissible, again demonstrating that $\Delta$ is $(2,2, t)$-permissible with a realization having a largest diameter at most 3 . Again note this argument holds for $t \geqslant 3$, similar to the colorings which end with $(\overline{8}, 1)$.

- $(2,1)$. By Lemmas 21 and 23 and since $t \geqslant 3, \Delta$ must end with type $(\overline{2}, 2,1)$. Then $\Delta$ ends with baabb. Note that each of the $(t-1)$ sets in $\mathcal{D}$ precedes $\{5 t-8,5 t-5\}$. Hence $\mathcal{D} \cup\{\{5 t-8,5 t-5\}\}$ is a set of $t$ permissible sets, which demonstrates that $\Delta$ is $(2,2, t)$-permissible with a realization having a largest diameter at most 3 . Again note this argument holds for $t \geqslant 3$, similar to the colorings which end with $(\overline{8}, 1)$ or $(5,1)$.

- $(2, \tau, 1)$. By Lemma 21 and since $t \geqslant 4, \Delta$ must end with type $(\overline{2}, \overline{2}, 2, \tau, 1)$. So $\Delta$ ends with baabaaab, baabbbbb, abbaaaab, or abbabbbb. Again note the second and third cases are not possible as the triples were not selected properly for its partition. In the first case, $\left.\Delta\right|_{[5 t-9]}$ ends with type $(\overline{2}, 1)$, or rather $(2,1),(5,1)$, or $(\overline{8}, 1)$.

Since $t-1 \geqslant 3$, we have $\left.\Delta\right|_{[5 t-9]}$ is therefore $(2,2, t-1)$-permissible with a permissible collection of $t$ pairs with largest diameter 4 ; let $\mathcal{D}^{\prime}$ denote this collection. So $\mathcal{D}^{\prime} \cup$ $\{\{5 t-8,5 t-4\}\}$ is a permissible collection of $t$ pairs, demonstrating the $(2,2, t)$ permissibility of $\Delta$ with a realization having a largest diameter at most 4 . In the fourth case, $\Delta$ is $(2,2, t)$-permissible with a realization having a largest diameter 1 by Lemma 22 .

We conclude with the observation that, in this work, we heavily use the binary nature of 2-colorings for constructing the AST partition used in our arguments. Hence we do not believe this method will translate nicely to $[r]$-colorings with $r \geqslant 3$ or the discovery of permissible $m$-sets with $m \geqslant 3$. 


\section{Acknowledgements}

We also thank our anonymous referee for their careful reading, detailed comments, and thoughtful suggestions for improving our paper.

\section{References}

[1] D. I. Bernstein, D. J. Grynkiewicz, and C. Yerger. On three sets with nondecreasing diameter. Discrete Math., 338(8):1328-1344, 2015.

[2] A. Bialostocki, P. Erdős, and H. Lefmann. Monochromatic and zero-sum sets of nondecreasing diameter. Discrete Math., 137(1-3):19-34, 1995.

[3] A. Bialostocki and R. Sabar. On constrained 2-partitions of monochromatic sets and generalizations in the sense of Erdös-Ginzburg-Ziv. Ars Combin., 76:277-286, 2005.

[4] A. Bialostocki and B. Wilson. On monochromatic sets of integers whose diameters form a monotone sequence. Integers, 9:A54, 717-723, 2009.

[5] B. Bollobás, P. Erdős, and G. Jin. Strictly ascending pairs and waves. In Graph theory, combinatorics, and algorithms, Vol. 1, 2 (Kalamazoo, MI, 1992), WileyIntersci. Publ., pages 83-95. Wiley, New York, 1995.

[6] P. Erdős, A. Ginzburg, and A. Ziv. Theorem in the additive number theory. Bull. Res. Counc. Israel Sect. F Math. Phys., 10F(1):41-43, 1961.

[7] D. Grynkiewicz and R. Sabar. Monochromatic and zero-sum sets of nondecreasing modified diameter. Electron. J. Combin., 13(1):\#R28, 2006.

[8] D. J. Grynkiewicz. On four colored sets with nondecreasing diameter and the ErdösGinzburg-Ziv theorem. J. Combin. Theory Ser. A, 100(1):44-60, 2002.

[9] D. J. Grynkiewicz. On four color monochromatic sets with nondecreasing diameter. Discrete Math., 290(2-3):165-171, 2005.

[10] A. Schultz. On a modification of a problem of Bialostocki, Erdös, and Lefmann. Discrete Math., 306(2):244-253, 2006.

[11] B. van der Waerden. Beweis einer Baudetschen Vermutung. Nieuw. Arch. Wisk., 15:212-216, 1927. 